\title{
PACIENTES PORTADORAS DE CÂNCER DE MAMA, SUBMETIDAS À RADIOTERAPIA COM ACELERADOR LINEAR E QUALIDADE DE VIDA
}

\author{
Lívia Almeida Fortunato \\ Graduanda do Curso de Enfermagem/ISECENSA/RJ \\ enf.liviafortunato@hotmail.com
}

Carolina Magalhães dos Santos

Doutora em ciências- IOC/ FIOCRUZ

carolmsantos@yahoo.com.br

\section{Anderson Pontes Morales}

Mestre em Ciências da Motricidade Humana - UCB/RJ

andersonmrl@hotmail.com

\section{Eduardo Viana Ricardo}

Mestre em Ciências da Educação - Universidade Americana/PY

enfeduardoviana@hotmail.com

\section{RESUMO}

A radioterapia é um tratamento eficaz contra o câncer de mama que tem a finalidade de destruir as células neoplásicas. Entretanto, pode produzir efeitos adversos causando alterações importantes na qualidade de vida (QV) das pacientes durante e após o tratamento. Assim, essa pesquisa que tem por objetivo comparar a qualidade de vida de mulheres com câncer de mama no início e após se submeterem a radioterapia com acelerador linear. $\mathrm{O}$ estudo foi realizado no hospital denominado OncoBeda, na cidade de Campos dos Goytacazes - RJ. A amostra foi composta por 20 pacientes do gênero feminino, com idade entre 33 a 87 anos. As mesmas responderam nos momentos pré e pós tratamento ao Instrumento WHOQOL-bref, composto por 26 questões, sendo 2 questões gerais sobre auto avaliação da QV e as outras 24 questões que envolvem 4 domínios, sendo eles "físico", "psicológico", "relações sociais" e "meio ambiente". Os resultados obtidos revelam que houve uma diferença ligeiramente significativa no domínio "físico", influenciando na avaliação geral da QV dessas pacientes. Sendo assim, conclui-se que mesmo não tendo um valor elevado de diferença entre os dois momentos avaliados, as pacientes apresentaram alteração na sua $\mathrm{QV}$ durante o tratamento.

Palavras-chave: Câncer de mama; Radioterapia; Qualidade de vida.

\begin{abstract}
Radiation therapy is an effective treatment against breast cancer aims to destroy cancer cells. However, could have adverse effects causing important changes in quality of life (QOL) of patients during and after treatment. Thus, this research aims to compare the quality of life of women with breast cancer at baseline and after undergo radiotherapy linear accelerator. The study was conducted at the hospital called OncoBeda in the city of Campos dos Goytacazes - RJ. The sample consisted of 20 patients were female, aged 33-87 years. The same account in the pre and post treatment to WHOQOL-BREF instrument, consisting of 26 questions, including 2 general questions about self-assessment of QOL and the other 24 questions involving four domains, namely "physical", "psychological", "social relations "and" environment. " The results show
\end{abstract}


that there was a slightly significant difference in "physical" domain, influencing the overall assessment of the QOL of these patients. Therefore, it is concluded that despite not having a high amount of difference between both times, the patients had a change in their QoL during treatment.

Keywords: Breast cancer; Radiotherapy; Quality of life.

\section{INTRODUÇÃO}

No Brasil, o câncer de mama é o mais comum destacando como a principal causa de morte entre as mulheres, além disso na década de noventa foi o mais frequente neste país. É o segundo tipo de câncer mais frequente no mundo sendo o primeiro entre as mulheres (TAVARES; TRAD, 2010).

A paciente acometida pelo câncer de mama e sua família, vivenciam o diagnóstico como um momento de intensa angústia, sofrimento e ansiedade. Identificado como uma das doenças mais temidas pelas mulheres devido à constância absurda com que vem ocorrendo e, principalmente pelos seus efeitos psicológicos que afetam a imagem pessoal e a sexualidade da mulher que o vivencia, consequentemente sendo devastadora tanto no físico como no psicológico (SOARES, 2009).

[...] o tratamento do câncer de mama pode envolver intervenções locais ou sistêmicas, utilizadas de forma independente ou concomitante. A cirurgia e a radioterapia podem ser entendidas como formas de tratamento local e que visam à remoção ou à destruição do tumor em uma determinada área do corpo (MAJEWSKI et al., 2012).

A conservação da mama, que tem como fundamentação a retirada do tumor através de cirurgia e no manejo axilar seguida de radioterapia, é hoje o tratamento local padrão para a doença em estádios iniciais, porém a escolha terapêutica vai depender das características anatomopatológicas, condições físicas da paciente, idade, estágio clínico e desejo da mesma. Um dos principais fatores prognóstico da doença é o diagnóstico precoce (MARTA et al., 2011).

[...] inicialmente, os equipamentos de radioterapia utilizavam fontes de rádio, as quais foram substituídas por fontes de césio-137 e de cobalto-60, depois que foi possível construí-las com alta atividade e de tamanho reduzido. Esse foi um grande avanço, pois, com uma atividade específica da fonte maior, foi possível aumentar a distância entre o equipamento e o paciente, além de ser mais prático não ter que trabalhar com fontes de rádio. Do ponto de vista tecnológico, o avanço seguinte veio com a construção dos aceleradores lineares, que permitiram utilizar terapeuticamente feixes de fótons e de elétrons com energias da ordem de megavolts (FURNARI, 2009).

Após cirurgias conservadoras, a radioterapia deve ser aplicada independente do tipo histológico, idade, uso de quimioterapia, hormonioterapia ou mesmo com margens cirúrgicas que estejam livres de comprometimento neoplásico. É utilizada com o objetivo destruir as células neoplásicas após a cirurgia ou para reduzir o tamanho do tumor antes de realiza-la (SCHETTINO et al., 2010).

No entanto, apesar de ser um tratamento eficaz, traz algumas manifestações clínicas agudas e crônicas, conhecidas como efeitos adversos. Entre eles, os principais são: as reações de pele (radiodermite, eritema), náuseas, mucosite, xerostomia, fadiga, anorexia, diarreia e disfagia (LEITE et al., 2013).

De acordo com Leite et al (2013) a consulta de enfermagem no setor de radioterapia é digna de atenção especial, pois o enfermeiro exerce essa atividade especificamente com mais frequência neste setor. $\mathrm{O}$

Persp. online: biol. \& saúde, Campos dos Goytacazes, 19 (5), 53-62, 2015

seer.perspectivasonline.com.br 
paciente procura pela consulta de enfermagem afim de adquirir informações para praticar o autocuidado e levar o tratamento adiante. A equipe de enfermagem deve servir de elo, atuando na educação de pacientes e familiares e na realização de cuidados específicos e como consequência, minimizando os efeitos adversos.

[...] a Qualidade de Vida (QV) é um conceito específico, envolve vários fatores na vida do indivíduo e pode ser considerada como um ótimo nível nas diversas funções (física, mental/cognitiva, emocional, social e funcional), incluindo também os relacionamentos, percepções de saúde, aptidão, satisfação com a vida, bemestar, e satisfação do paciente com o tratamento, resultados, estado de saúde e perspectivas futuras (NICOLUSSI; SAWADA, 2011).

A QV se dá a partir de uma avaliação subjetiva dos processos da doença e da recuperação. Profissionais e pacientes tentam identificar a interferência do tratamento oncológico para o controle ou a cura da doença, buscando entender os desfechos do tratamento bem-sucedido na compreensão da própria paciente. Espera-se que medidas quantitativas de QV possam auxiliar na definição de ações que deem prioridade a promoção da saúde e possam guiar estratégias de intervenções terapêuticas (MAJEWSKI et al., 2012).

Este trabalho tem por objetivo comparar a qualidade de vida de mulheres com câncer de mama no início e após submeterem-se a radioterapia com acelerador linear.

\section{METODOLOGIA}

\subsection{Tipo do Estudo}

Trata-se de uma pesquisa quantitativa que para Leopardi (2002) essa abordagem é utilizada quando há a preocupação de se garantir a objetividade e credibilidade dos achados, onde a questão proposta indica uma atenção com a quantificação, com a comparação de eventos ou a replicação de estudos.

\subsection{Local do estudo}

O presente estudo foi realizado na unidade de tratamento ao câncer denominada Oncobeda, pertencente à rede de prestação de serviço privado em saúde designada como: Hospital Geral Dr. Beda. Esta instituição está situada à rua Saldanha Marinho, 422, Centro, Campos dos Goytacazes - RJ. O referido hospital foi escolhido por ser referência em casos de oncologia.

\subsection{Amostra}

A amostra foi composta por 20 pacientes do gênero feminino, com idade entre 33 a 87 anos. Os critérios de inclusão para seleção dos sujeitos da pesquisa foram pacientes do gênero feminino, portadoras de câncer de mama que se submeteram ao tratamento de radioterapia com acelerador linear, enquanto os critérios de exclusão foram pacientes não alfabetizados e que não queiram assinar o Termo de Consentimento Livre e Esclarecido (TCLE).

\subsection{Instrumento de Pesquisa}

Para coleta de dados nesse estudo, utilizamos o instrumento WHOQOL-bref (apêndice 1). O mesmo foi testado em várias culturas, tendo sido validado, inclusive, para o Brasil. O instrumento WHOQOL-bref considera os últimos quinze dias vividos pelos respondentes. O mesmo é composto por vinte e seis questões: Duas são gerais, sendo que uma se refere à VIDA e a outra à SAÚDE e não estão incluídas nas equações estabelecidas para análise dos resultados (FLECK et al, 2000, p.180) 
As demais vinte e quatro perguntas são relativas a quatro domínios e suas respectivas facetas, como segue: Domínio I - físico, focalizando as seguintes facetas: dor e desconforto, energia e fadiga, sono e repouso, atividades da vida cotidiana, dependência de medicação ou de tratamentos, capacidade de trabalho; Domínio II - psicológico, cujas facetas são: sentimentos positivos, pensar, aprender, memória e concentração, autoestima, imagem corporal e aparência, sentimentos negativos, espiritualidade, religiosidade e crenças pessoais; Domínio III - relações sociais, que inclui as facetas a seguir: relações pessoais, suporte (apoio) social, atividade sexual; Domínio IV - meio ambiente, abordando as facetas: segurança física e proteção, ambiente no lar, recursos financeiros, cuidados de saúde e sociais: disponibilidade e qualidade, oportunidades de adquirir novas informações e habilidades, participação em, e oportunidades de recreação/lazer, ambiente físico: poluição, ruído, trânsito, clima, transporte (FLECK et al, 2000, p.182).

O período da coleta de dados foi de quatro meses (julho a novembro de 2015). Cada sujeito respondeu em dois momentos o mesmo formulário: no momento pré (início) e outro no momento pós (final) do tratamento. Para identificar a elegibilidade das pacientes para os referidos momentos de avaliação, foi feito uma consulta aos prontuários das mesmas. Os formulários foram aplicados pelos pesquisadores respeitando a vontade dos sujeitos da pesquisa, que elegeram um momento oportuno para aplicação dos mesmos.

\subsection{Análise Estatística}

O procedimento de pontuação do WHOQOL-Bref foi efetuado informaticamente através de uma planilha de excell elaborada por Pedroso et al. (2010). Após a referida pontuação, passou-se ao tratamento dos dados através da análise estatística descritiva e inferencial. Testou-se a normalidade da amostra em estudo através do teste estatístico de Shapiro-Wilk, tendo-se constatado que a amostra do estudo em alguns domínios avaliados não segue uma distribuição normal. Entre as técnicas estatísticas utilizadas salientam-se as média aritmética $(\bar{x})$ e mediana (Me). Foram ainda utilizadas medidas de dispersão, nomeadamente, o desvio padrão (s) e o desvio interquatílico (dq). Para avaliar as diferenças entre os momentos pré (início) e pós (fim) tratamento foi utilizado o teste o Mann Whitney para amostras pareadas e não paramétricas. Já para as amostras pareadas foi utilizada o teste "t" de Student para amostras paramétricas. Em todas as análises, adotou-se o nível de significância estatística de 5\% ( $\mathrm{p}<0,05)$. Os dados oriundos dos procedimentos descritos acima foram analisados pelo software 5.0 GraphPad Prism ${ }^{\circledR}$ versão 5.0 (Graphpad Software, Inc., www.graphpad.com).

\subsection{Aspectos Éticos da Pesquisa}

O projeto foi submetido ao Comitê de ética e Pesquisa (CEP) do Instituto Superior de Ensino do Censa (ISECENSA), cumprindo as especificações da resolução 466/12 do ministério da saúde, e foi aprovado sob o número de CAAE: 38608514.9.0000.5524, no dia 28/11/2014.

Essa pesquisa não acarretará ônus financeiro para os sujeitos participantes, ficando a cargo dos pesquisadores as despesas com o desenvolvimento da mesma. O risco para as pacientes ao participar da amostra será um possível constrangimento. Não haverá, dentro da metodologia aplicada, aspectos que possam acarretar prejuízos de ordem biológica ou psicossocial aos sujeitos da pesquisa ou aos que se encontram sob sua responsabilidade.

Quanto ao instrumento, esse foi aplicado de forma conveniente aos sujeitos e respeitando seus limites. Os sujeitos da pesquisa receberam um termo de consentimento livre e esclarecido, que foi a permissão do sujeito da pesquisa e/ou de seu representante legal. Após explicação completa e pormenorizada sobre a natureza da pesquisa, seus objetivos, métodos, benefícios previstos, potenciais riscos e o incômodo que esta possa acarretar, formulada em um termo de consentimento, autorizando sua participação voluntária na pesquisa, de acordo com a resolução 466/12. Aos possíveis sujeitos da pesquisa foi garantido o anonimato, quando utilizado para fins de produção acadêmica e de divulgação científica e direito de se retirar da pesquisa a qualquer momento.

Persp. online: biol. \& saúde, Campos dos Goytacazes, 19 (5), 53-62, 2015

seer.perspectivasonline.com.br 


\section{RESULTADOS E DISCUSSÃO}

\section{Domínio: Físico}

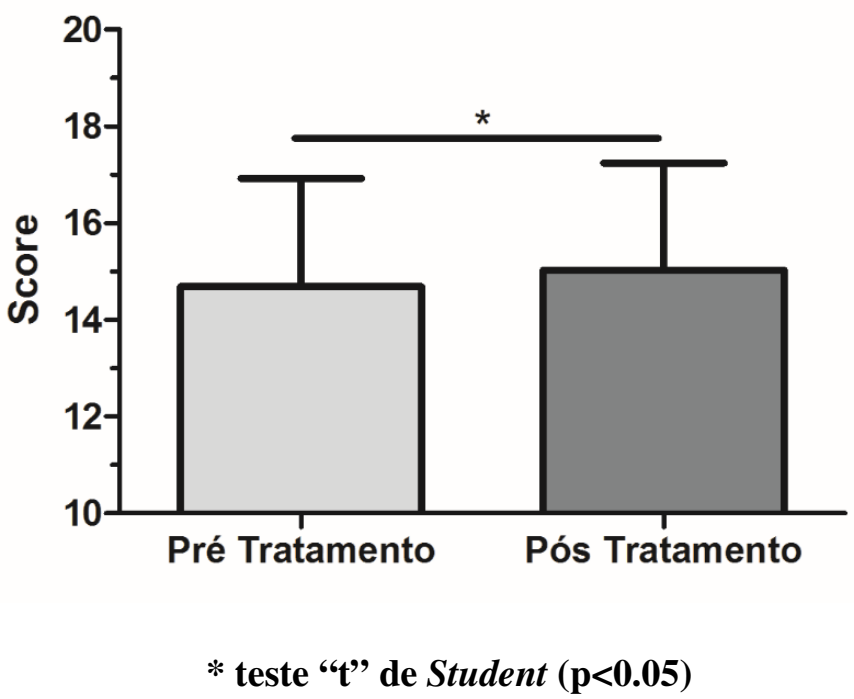

Figura 1. Resultados das inferências realizadas entre os momentos pré e pós tratamentos. Valores expressos em Média \pm Desvio padrão.

A figura 1 representa os escores obtidos nos momentos pré $(14.69 \pm 2.24)$ e pós $(15.03 \pm 2.21)$ tratamento em relação ao domínio "físico". Houve diferenças significativas entre os momentos pré e pós tratamento $(\mathrm{p}<0.05)$.

Observamos que houve diferenças positivas significativas entre os momentos pré e pós tratamento no domínio físico, o que corrobora com Bantema-Joppe (2015) relatando que as melhorias observadas ao longo do tempo na QV global, especialmente para as mulheres jovens, são provavelmente o resultado de vários mecanismos. Uma verdadeira melhoria ao longo do tempo, com menos queixas físicas, podem estar presentes após a tensão inicial de diagnóstico e tratamento de primeira fase. Outro estudo, realizado por Alegrance et al (2010) foi relatado que a função física das participantes são satisfatórias e que a reabilitação física é bastante favorável à melhora desses sintomas. A prática de exercícios físicos globais melhorou a função física, saúde geral e vitalidade de mulheres com câncer de mama. Por isso, tanto o estímulo à prática de atividade física quanto os exercícios terapêuticos se fazem necessários à reabilitação do tratamento póscâncer de mama. 


\section{Domínio: Psicológico}

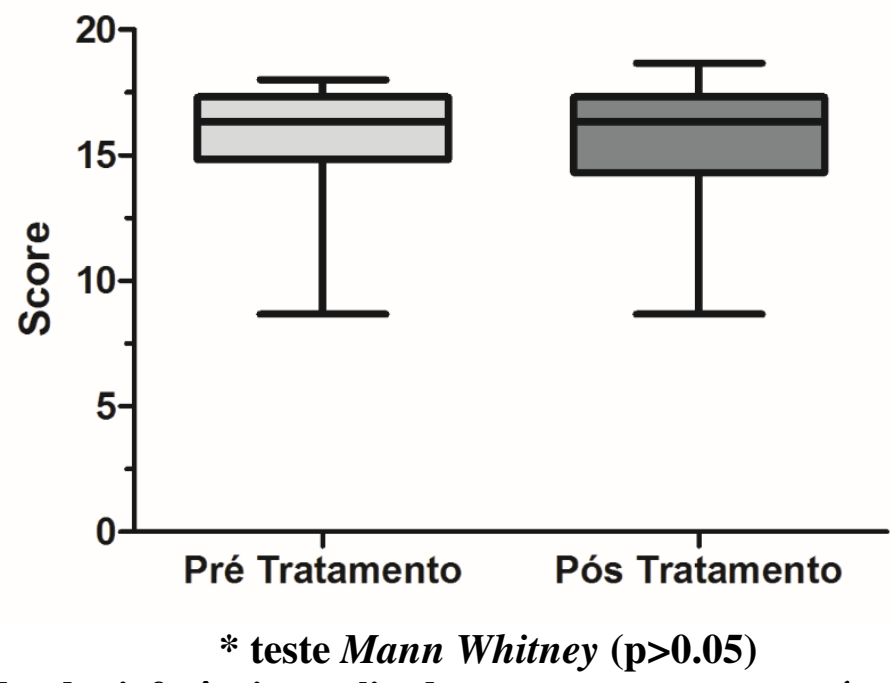

Figura 2. Resultados das inferências realizadas entre os momentos pré e pós tratamento.Valores expressos em Mediana (Desvio Interquatílico)

A figura 2 representa os escores obtidos nos momentos pré 16.34 (2.16) e pós 16.33 (2.33) tratamento em relação ao domínio "psicológico". Não houve diferenças significativas entre os momentos pré e pós tratamento $(\mathrm{p}>0.05)$.

\section{Domínio: Relações Sociais}

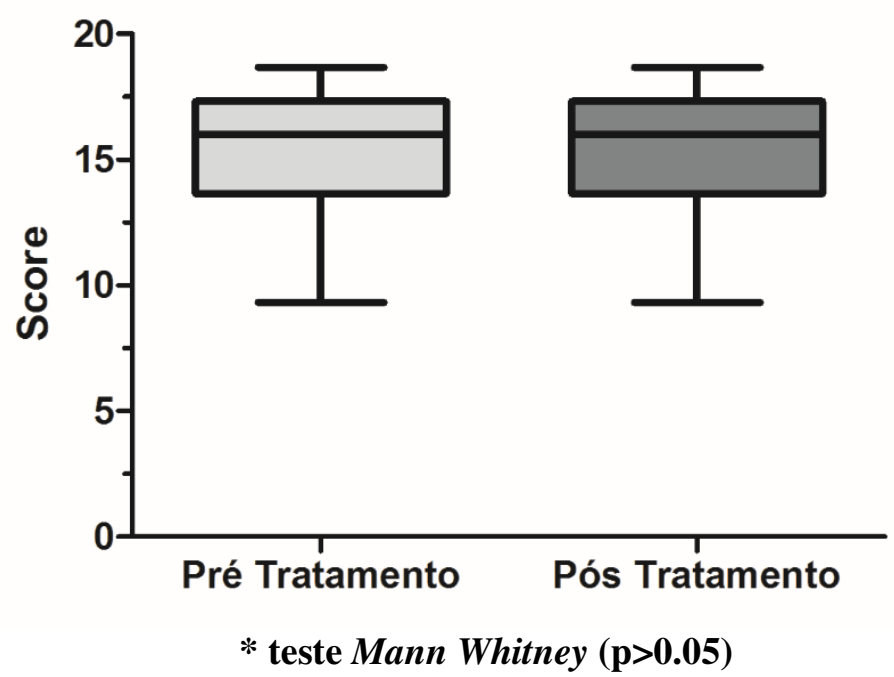

Figura 3. Resultados das inferências realizadas entre os momentos pré e pós tratamento.Valores expressos em Mediana (Desvio Interquatílico)

A figura 3 representa os escores obtidos nos momentos pré 16.00 (2.99) e pós 16.00 (2.99) tratamento em relação ao domínio "relações sociais". Não houve diferenças significativas entre os momentos pré e pós tratamento $(\mathrm{p}>0.05)$. 
No presente estudo não houve diferença significativa no domínio relações sociais, porém no estudo realizado por Vendrusculo (2011) os escores obtidos, relativo a função social, relacionada a QV, evidenciam que as mulheres com câncer de mama apresentam um comprometimento moderado neste domínio; desta forma a capacidade de interação no contexto em que estão inseridas, representadas pelo desempenho das atividades de lazer, trabalho e relações familiares, mostra-se comprometida. Corroborando com Monsanto (2013) que verificou uma diminuição na subescala função social, que poderá ser resultado do isolamento dos doentes nos seus relacionamentos sociais, após diagnóstico e tratamentos associados à doença.

\section{Domínio: Meio Ambiente}

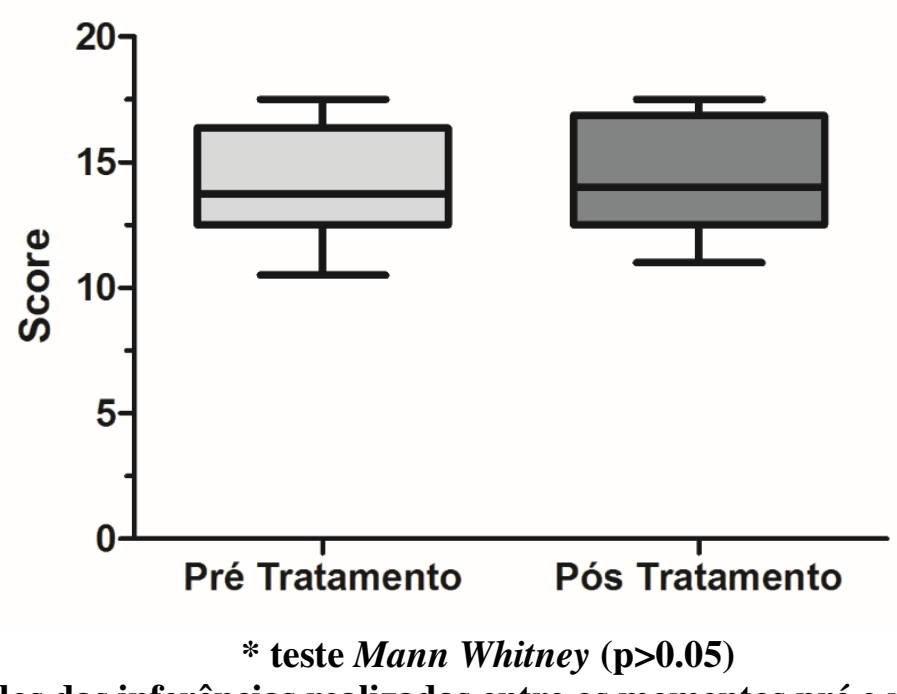

Figura 4. Resultados das inferências realizadas entre os momentos pré e pós tratamento.Valores expressos em Mediana (Desvio Interquatílico).

A figura 4 representa os escores obtidos nos momentos pré 13.75 (3.62) e pós 14.00 (4.12) tratamento em relação ao domínio "meio ambiente". Não houve diferenças significativas entre os momentos pré e pós tratamento $(\mathrm{p}>0.05)$. 


\section{Domínio: Auto Avaliação da QV}

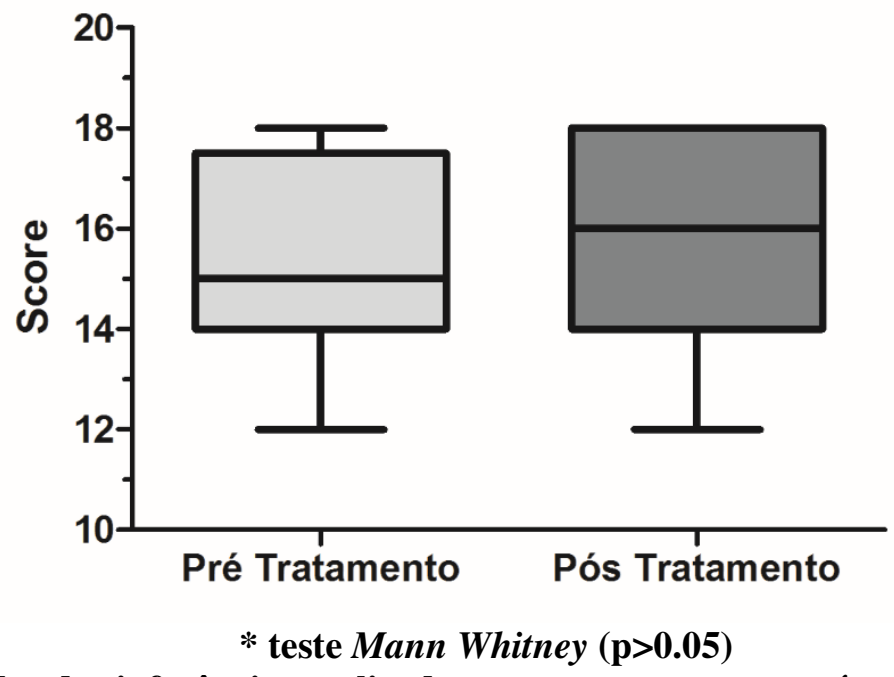

Figura 5. Resultados das inferências realizadas entre os momentos pré e pós tratamento.Valores expressos em Mediana (Desvio Interquatílico)

A figura 5 representa os escores obtidos nos momentos pré 15.00 (2.50) e pós 16.00 (4.00) tratamento em relação ao domínio "auto avaliação da QV". Não houve diferenças significativas entre os momentos pré e pós tratamento $(\mathrm{p}>0.05)$.

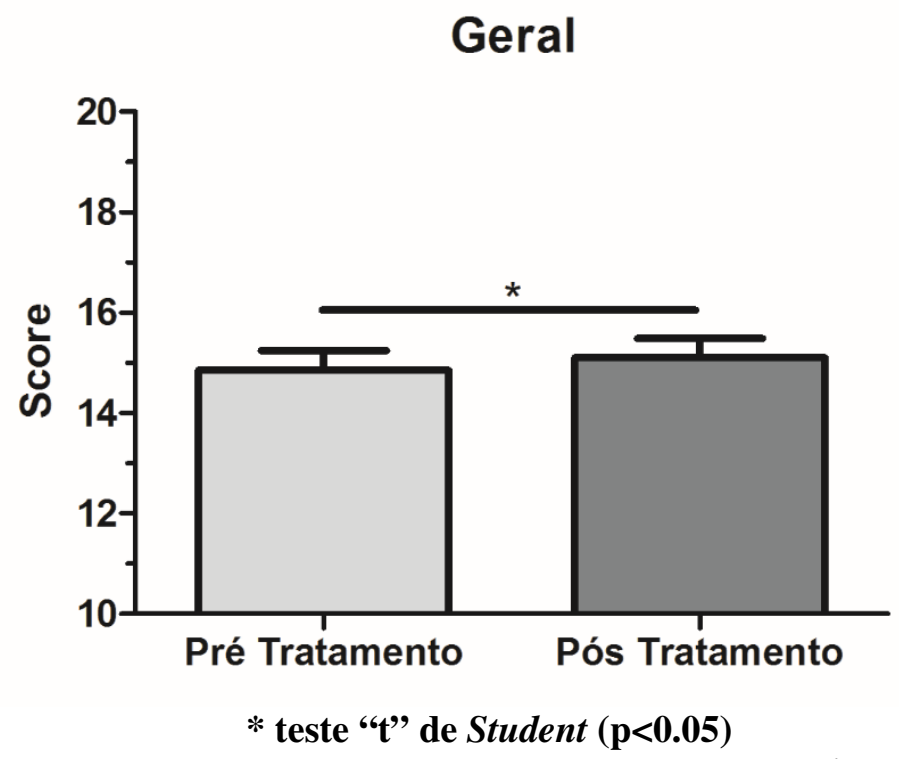

Figura 6. Resultados das inferências realizadas entre os momentos pré e pós tratamentos. Valores expressos em Média \pm Desvio padrão.

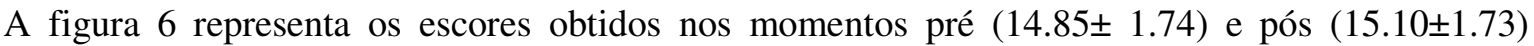
tratamento em relação ao domínio "geral". Houve diferenças significativas entre os momentos pré e pós tratamento $(\mathrm{p}<0.05)$. 
De acordo com os resultados avaliados, observamos que a QV geral dessas pacientes foram ligeiramente influenciadas pelo tratamento radioterápico. No estudo realizado por Monsanto (2013) verificou-se que no final do tratamento houve um aumento da função emocional, o que poderá estar associado à relação estabelecida entre os doentes e os profissionais de saúde, nomeadamente ao nível do aumento da autoestima. Esta relação poderá influenciar positivamente a qualidade de vida destes doentes. Outro estudo realizado por Vendrusculo (2011), foi observado que poucas relataram a presença dos efeitos adversos, o que pode ter acontecido por se encontrarem em um período de finalização ou já terem terminado o tratamento, quando a manifestação de alguns desses efeitos pode estar diminuída ou não mais se apresentar. Leite (2013) considera que a utilização do diagnóstico de enfermagem pode favorecer a autonomia do enfermeiro, pois serve de referência para o desenvolvimento das intervenções de enfermagem, possibilitando o exercício do raciocínio crítico e julgamento clínico do enfermeiro. O referido autor ainda relata, que o respeito à individualidade do paciente é pontuado, sendo destacado que o cuidado individualizado articula uma relação favorável com a equipe multiprofissional, paciente e família, favorecendo a humanização da assistência. A radioterapia é uma modalidade terapêutica muito utilizada no tratamento do câncer, mas que costuma acarretar aos pacientes alguns efeitos adversos, no entanto o enfermeiro atua nesse setor na tentativa de minimizar esses efeitos.

\section{4- CONCLUSÃO}

A partir dos resultados obtidos, observamos que nos domínios "Psicológico", "Relações Sociais", "Meio Ambiente", e "Auto Avaliação da qualidade de vida (QV)" não apresentaram diferenças significativas entre os momentos pré e pós tratamento. No entanto, evidenciamos que houve uma diferença ligeiramente significativa no domínio "físico", influenciando na avaliação geral da QV dessas pacientes. Ao verificar uma melhoria no domínio físico, entendemos que este pode estar também relacionado com o resultado do apoio e orientação feita pela equipe multidisciplinar deste referido hospital, principalmente pela equipe de enfermagem que atua nos cuidados específicos individuais e na educação dessas pacientes e familiares, minimizando os efeitos colaterais causados pela radiação.

Sendo assim, concluir-se que mesmo não tendo um valor elevado de diferença entre os momentos pré e pós tratamento de radioterapia com acelerador linear em todos os domínios, as pacientes apresentaram alteração positiva na sua QV. Acredita-se que através de práticas educativas e desenvolvidas, implementadas e supervisionadas pelo enfermeiro, se pode melhorar a qualidade de vida e ao tratamento de câncer de mama, tornando assim útil a pesquisa para a sociedade, profissionais e estudantes que estão envolvidos neste contexto direto e indiretamente. O instrumento WHOQOL-Bref avalia um curto tempo, sendo os últimos quinze dias vivido pelo indivíduo. Talvez para observar maiores diferenças na QV, seja necessário um tempo mais longo de observação após o tratamento.

\section{REFERÊNCIAS}

ALEGRANCE, F. C.; SOUZA, C. B.; MAZZEI, R. L. Qualidade de vida e estratégias de enfrentamento em mulheres com e sem linfedema pós-câncer de mama. Revista Brasileira de Cancerologia, v. 56, n. 3, p. 341-351, 2010.

BANTEMA-JOPPE, E. J.; BOCK, G. H.; WOLTMAN, M.; BUSZ, D. M.; RANCHOR, A. V.; LANGENDIJK J. A.; MADURO J. H.; HEUVE, E. R., The impact of age on changes in quality of life among breast cancer survivors treated with breast-conserving surgery and radiotherapy. British Journal of Cancer. v. 112, p.643., 2015.

FLECK, M.P.A.; LOUZADA, S.; XAVIER, M.; CHACHAMOVICH, E.; VIEIRA, G.; SANTOS, L. Aplicação da versão em português do instrumento abreviado de avaliação da qualidade de vida "WHOQOL - bref”. Rev. Saúde Pública, São Paulo, v. 34, n. 2, Apr. 2000

Persp. online: biol. \& saúde, Campos dos Goytacazes, 19 (5), 53-62, 2015

seer.perspectivasonline.com.br 
FURNARI, L. Controle de Qualidade em Radioterapia. Revista Brasileira de Física Médica. v. 3, n.1, p. 77-90, 2009.

LEITE, F. M. C.; FERREIRA, F. M.; CRUZ, M. S. A.; LIMA, E. F. A.; PRIMO, C. C. Diagnósticos de enfermagem relacionados aos efeitos adversos da radioterapia. REME - Rev Min Enferm. V. 17, n. 4, p. 940-945, 2013.

LEOPARDI, M.T. Metodologia da pesquisa em saúde. UFSC/Pós graduação em Enfermagem Florianópolis, $2^{\mathrm{a}}$ ed. p. 290, 2002.

MAJEWSKI, J. M. et al. Qualidade de vida em mulheres submetidas à mastectomia comparada com aquelas que se submeteram à cirurgia conservadora: uma revisão de literatura. Ciência \& Saúde Coletiva, v. 17, n. 3, p 707-716, 2012.

MARTA, G. N. et. al. Câncer de mama estádio inicial e radioterapia: atualização. Rev Assoc Med Bras. v. 57, n.4, p. 468-474, 2011.

MOnSANTO, F.; LANÇA, C.; SÁ, A. C.; COELHO, C. M.; CAROLINO, E. Influência do tratamento de radioterapia na qualidade de vida dos doentes com cancro de mama. Saúde e Tecnologia. V. 9, p. 43, 2013.

NICOLUSSI, A C.; SAWADA, N O. Qualidade de vida de pacientes com câncer de mama em terapia adjuvante. Revista Gaúcha Enfermagem. Porto Alegre (RS). v. 32, n. 4, p. 759-766, dez 2011.

PEDROSO, B. et al. Cálculo dos escores e estatística descritiva do WHOQOL-bref através do Microsoft Excel. Revista Brasileira de Qualidade de Vida, Ponta Grossa, v. 2, n. 1, p. 31-36, jan./jun. 2010.

SCHETTINO, R. C. et. al. Função pulmonar em mulheres com câncer de mama submetidas à radioterapia: um estudo piloto. Fisioterapia e Pesquisa, São Paulo, v.17, n.3, p.248-52, jul/set. 2010.

SOARES, R. G. Aspectos emocionais do câncer de Mama. Sociedade brasileira de psico-oncologia boletim eletrônico sbpo. Ano IV, ed. 3, jul-ago-set 2009.

TAVARES, J. S. A.; TRAD, L A B. Estratégias de enfrentamento do câncer de mama: um estudo de caso com famílias de mulheres mastectomizadas. Ciência e Saúde Coletiva, v. 15, n. 1, p. 1349-1358, 2010.

VENDRUSCULO, L. M. Capacidade funcional e qualidade de vida de mulheres com câncer após tratamento oncológico. 2011. 95p. Dissertação (Mestrado) - Escola de Enfermagem de Ribeirão Preto, Universidade de São Paulo, São Paulo, 2011). 